\title{
Non-destructive Assessment of Relief Marking Parameters of Heat Shrinkable Installation Parts for Aviation Technology
}

\author{
Aleksandr P. Kondratov ${ }^{1,}{ }^{*}$, Mariya $V$. Konovalova ${ }^{1}$, Egor P. Cherkasov ${ }^{1}$ and Irina A. Savenkova ${ }^{2}$ \\ ${ }^{1}$ Moscow Polytechnic University, 107023 Moscow, Russia \\ ${ }^{2}$ National Research University Higher School of Economics, 101000 Moscow, Russia
}

\begin{abstract}
The article explains a new method of relief marking of heat-shrinkable tubing and sleeves made of polymer materials with "shape memory effect." Method of instrument evaluation of relief marking stereometry of installation parts for aviation equipment, made of polyvinyl chloride, polyethyleneterephthalate and polystyrene was developed and the results were explained. Parameters of pin-point relief marking and compliance of point forms to the Braille font standard were determined with the use of the non-destructive method based on the color of interference pattern with precision of $0.02 \mathrm{~mm}$.
\end{abstract}

\section{Introduction}

For new samples of complex machinery [1] in aviation industry and aircraft-repair works $[2,3]$, polymer heatshrinking tubes and sleeves are used with «shape memory» in order to provide additional mechanical protection, sealing action and electrical insulation of contact, complex connections of electrical wires etc.

Various types of marking are used in order to control technical conditions of various aviation components and determining the appurtenance of communication tools. As a rule, multicolored isolation cable sheaths, tags and flags are used for the purpose of identification. In the aircraft electrical systems all wires, harnesses and cables are subject to marking [4]. Appurtenance of wires to various systems and feeders is determined not only by wire insulation color and by marking, that presents an alphanumeric code, which is put on polymer insulation of the wire or on its identification tag. In the absence of direct access, insufficient visibility or bad lighting conditions of the communication location it is feasible to use relief marking.

In order to make the process of reading the printed information on the insulating jacket or sleeve, that is, for example, carried out with the usage of Braille, relief symbols should have a specific appearance, specific location on the plane and comply with strictly specified stereometry parameters [5].

The technology of applying the relief tags on the products made of heat-shrinking materials $[6,7]$ that was developed by us allows to record information on soft and flexible polymer films with compliance of allowable sizes of Braille font symbols. In order to determine the optimum conditions and control the process of product tagging (labels, packaging, couplings and tubes) made from polymer materials, instrumental quantitative evaluation of micro-size relief stereometry is required.
There are a few methods of analyzing the dimensional transparent microscopic objects which are used in medicine, forensics, materials science $[8,9]$.

In previous experimental works $[10,11]$ measurement of parameters of tactile relief was carried out in the following way. Sample of elastic label product was cooled up to the temperature of below the polymer glass-formation in order to increase the rigidity and was dissected with curring blade along the relief crest. Afterwards the received cut-section was photographed dimensions of the image were measured and evaluated, with the usage of gauge-metering function of optical digital USB-microscope. At the stage of sample dissection deformation of the tagg's relief is unavoidable, consequence of which is the measurement error in the amount of $+0,3$ micron.

\section{Goals and objectives}

Development of a high precision instrumental procedure of non-contacting evaluation of stereometry of relief marking of flexible and elastic polymer parts with «shape memory», that are applied during installation and repair of aviation unit communications.

\section{Materials and methods}

Heat-shrink tubing and polymer films of industrial production from polyvinyl chloride, polyethylene terephthalate and polystyrene, which thickness is 50 microns. Producers of heat-shrinkable materials are Dongil Chemical and Alfterm. Coefficient of maximum shrinkage during $\mathrm{T}=90 \pm 5^{\circ} \mathrm{C}$ heating should be around $70 \%$. Relief point-like and linear signs should be applied on the samples of polymer films. Point-like tagging was carried out with the usage of soldering station that provides temperature control at $100 \pm 1^{\circ} \mathrm{C}$ of cylindrical

* Corresponding author: apkrezerv@,mail.ru 
soldering iron rod with a flat foundation and diameter of 1.0 and $3.0 \mathrm{~mm}$. Linear tagging was carried out with the usage of impulse heating unit with local thermal processing [12]. «Developing» of relief was carried out with settlement on holding base (mounting jig) that imitated the isolated communication line of aviation unit through heating with a hot air flow or in water $\left(85-95^{\circ} \mathrm{C}\right)$ by the special method [10].

\section{Results and discussion}

Algorithm of the new method of relief marking of heat shrinkable parts made of thermoplastic polymers includes the following operations:

Local thermal processing of samples (heat-shrinkable tubing or film) in isometric constraints, application of the sample on a rigid base (communication tools, wiring plugs) and heat shrinking process in some hot heattransfer medium (air, water).

Local thermal processing of the product with «shape memory» is carried out in isometric conditions. During local thermal processing of the small product section the isometric mode is provided by the hardness of the polymer material that surrounds the area of local polymer heating. As a result of hardness of glass-like polymer the shrinkage of the heat-treated part doesn't take place, instead local relaxation process of internal stresses happens. Stabilization of geometrical dimensions of the local thermal processing area is a result of relaxation of the internal stresses, which means the loss of ability for heat-shrinking.

During heating of the product after local heattreatment process through submerging the sample into the hot medium its surface drastically reduces. Areas of local thermal processing do not change their dimensions of thermostabilized area and thus it bulges out. Relief is formed, that contains visual and tactile information. Relief can have the form of vectors or geometric shapes or form of dots with the stereometry of Braille font. Main factors that determine the form and dimensions of the relief are shrinking properties of the polymer (coefficient of thermal shrinkage), dimensions of the areas of local thermal processing (diameter of the dot foundation or width of lines) or ratio of heat-shrinkable tube's (sleeve) dimensions and dimensions of communication tools where the enclosure or sleeve are applied.

Types of relief labeling on heat-shrinkable pipes that are applied in electrical and electronic communication tools of aviation units are shown as a scheme on the Fig. 1 and 2. Non-contact composition of 3D models of relief of the experimental sample was used for instrument evaluation of stereometry of aviation equipment installation parts surfaces made of transparent polymer materials. Laboratory direct microscope of reflected light Carl Zeiss Axio Lab.A1, equipped with additional measuring device PhaseView, was used for this purpose. Determination of the distance to the fragment of the surface was carried out through analyzing the interference pattern that is formed inside the additional measuring device. This device contains calibrated emitting source, which allows measuring the precise dimensions of the relief with use of results of preliminary measurement of the distance to the researched sample based on location of maximum points and minimum points of interference pattern. Geometric data of the optical path that are contained in calibration files of the used software are used for preliminary measurement of the distance.

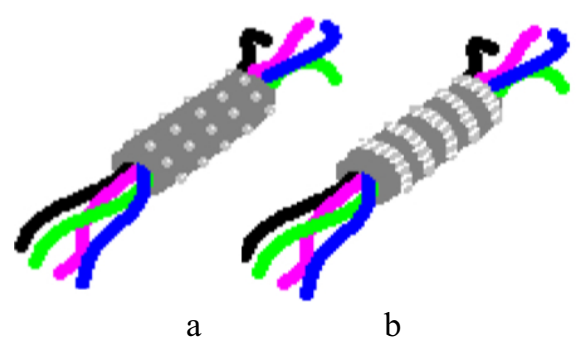

Fig. 1. Type of sections with the relief labeling of the products with «shape memory» from heat shrinkable shelter of multicore cable: a) pinpoint marking; b) linear marking

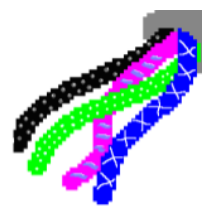

Fig. 2. Types of section with relief labeling of separate wires made from heat shrinkable tubes

In order to carry out the optical tests we have to cover the surface of the polymer tube sample or film with pigment or dye solution of any dark color, because of distortion-noises, which occur on the projections of transparent objects. Coloration of the surfaces of researched objects was carried out with the use of inks that contain ethanol.

Building of 3D model is provided by GetPhase software. The relief of the surface looks much more informative in isometrics (Fig. 3). The height of characteristic points of the surface relief is based on the color tone range (shown on the right on the Fig. 3a, b).

Quantitative characteristics of the relief that were received during measurement of the relief marking of elements of various types are given in the Table 1 and Table 2. Geometry of the marking [13] is not very varied, it can either be pin-point or linear, but the dimensions of the marking can change depending on the area of processed polymer surface.

Table 1 contains the information of overall dimensions of pin-point marking of polymers with different chemical composition, but with the same thickness. From the table it is clear, that the more a diameter of the heated element is, the higher each point becomes. Table 2 presents results of the amplitude and period measurements of the relief marking. As a result, because of the fact that polyethylenterephtalate has higher hardness, the relief that was formed by pin-point and linear marking on these films samples is quite high in comparison with relief on other films, used in the experiment. 


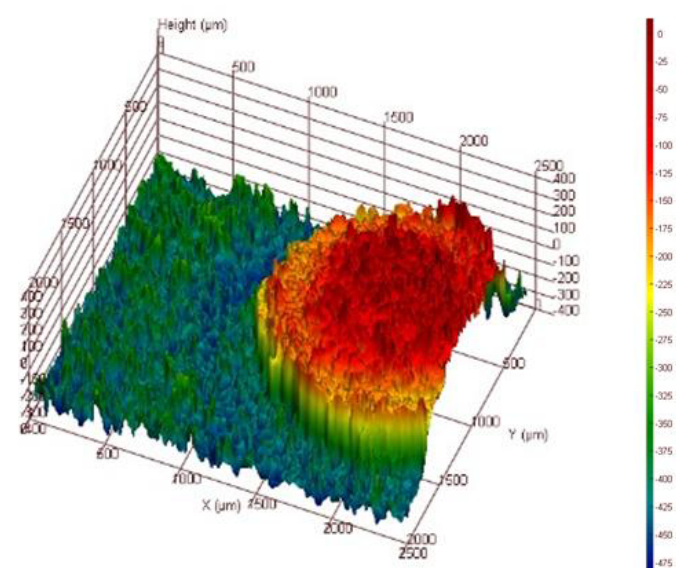

$\mathrm{a}$

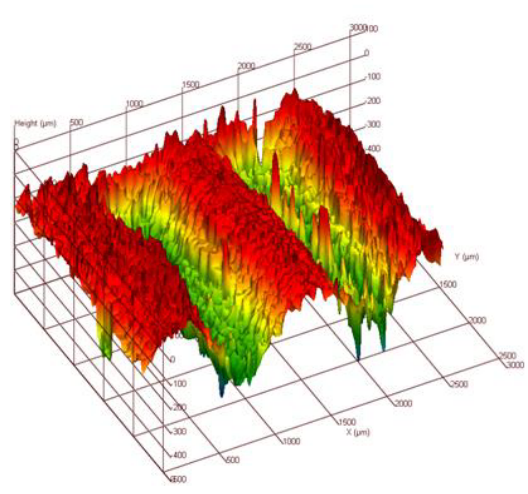

b

Fig 3. The 3D-Model of fragments of various relief marking of polymer films: a) Pinpoint marking; b) Linear marking

Table 1. Parameters of pin-point marking of the heat-shrinkable polymer samples

\begin{tabular}{|c|c|c|}
\hline \multirow{2}{*}{ Polymer material } & \multicolumn{2}{|c|}{ Height of the point, $\mu \mathrm{m}$} \\
\cline { 2 - 3 } & $\begin{array}{c}\text { Diameter of } \\
\text { heated element } \\
-3.0 \mathrm{~mm}\end{array}$ & $\begin{array}{c}\text { Diameter of } \\
\text { heated element } \\
-1.0 \mathrm{~mm}\end{array}$ \\
\hline Polyvinyl chloride & 442 & 370 \\
\hline Polystyrene & 563 & 243 \\
\hline Polyethyleneterephthalate & 667 & 30 \\
\hline
\end{tabular}

Table 2. Parameters of the linear marking of the polymer film with the thickness of $50 \mu \mathrm{m}$

\begin{tabular}{|l|l|l|}
\hline \multirow{2}{*}{ Polymer material } & \multicolumn{2}{|c|}{ Parameters } \\
\cline { 2 - 3 } & \multicolumn{1}{|c|}{$\begin{array}{c}\text { Amplitude, } \\
\mu \mathrm{m}\end{array}$} & Period, $\mu \mathrm{m}$ \\
\hline Polyvinyl chloride & 264 & 1180 \\
\hline Polystyrene & 462 & 1660 \\
\hline Polyethyleneterephthalate & 512 & 1720 \\
\hline
\end{tabular}

\section{Conclusion}

The quality of visual control and preventive measures against shutdowns of electrical networks in aviation equipment very much depends on the quick identification of communication tools (wires, cables, tags and flags). Their identification is based on the color differences or quick finding and reading the printed markings, what in conditions of bad visibility and limited access to the units of aviation equipment can be quite challenging. Thus, the presence of relief symbols on heat shrinkable tubes will considerably simplify the process of identifying the communication linkages.

Additionally, the developed instrumental method of the non-contact evaluation of stereometry of relief marking of felxible and elastic polymers allows carrying out more detailed studying of the investigated samples' surfaces. Without breaking the integrity of the sample, measurements were carried out with high precision and high accuracy.

\section{References}

1. V.A. Kaniskin, Operation of power electric cables: Teaching guide (Saint-Petersburg, PEIPK, 2001)

2. N.M. Ilyshin, Methodical recommendations on organizational maintenance and first line repair of electrical systems of aircraft vehicles (Lyubertsy: 13 GNII RF Ministry of Defence, 1985)

3. Airplane Il-76 /Battle Damage Repair Manuals/ Section Aircraft Electrical System (LLC "AviaMedia", 2004-2007)

4. Braznburg, E.Z. et al. Cables iwht plastic insulation and couplings for their installation (Moscow, Energoatomizdat, 1987)

5. Marburg Medium spacing convention for braille. Website of the company Deutsche Blindenstudienanstalt e. V Braille-Druckere Postbox

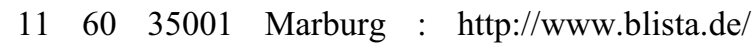
download/druckerei/braille-dimensions.

6. M.V. Konovalova, A.P. Kondratov, The patent of the Russian Federation № 150223 (2015)

7. A.P. Kondratov, Izvestia vuzov. Problem of the graphic arts and publishing, 4, 57-65 (2010)

8. Methods of research in histology, cytology and embryology. http://profmed.blogspot.ru/2013/04 /blog-post_28.html. Date of access 03.03.2016

9. V.B. Konstantinov, V.A. Babenko, Applied physics journal, 77 (2007)

10. M.V. Konovalova, A.P. Kondratov, Izvestia vuzov. Problem of the graphic arts and publishing, 3, 53-59 (2015)

11. A.P. Kondratov, Modern Applied Science; 8(6), 204-209 (2015)

12. M.V. Konovalova, A.P. Kondratov, V.G. Nazarov, A.F. Benda, 46th Annual International Conference on Graphic Arts and Media Technology Managem Management and Education, Athens and Corinthia, Greece, p. 251 (2014)

13. E.P. Cherkasov, A.P. Kondratov Izvestia vuzov. Problem of the graphic arts and publishing, 1, 43-47 (2015) 